Pesq. Vet. Bras. 36(3):216-220, março 2016

DOI: $10.1590 / \mathrm{S} 0100-736 \mathrm{X} 2016000300011$

\title{
Análise histomorfométrica dos cardiomiócitos e deposição de colágeno no músculo cardíaco de ratas ooforectomizadas ${ }^{1}$
}

\author{
Maria C.R. Silva², Maria J.G. Arandas ${ }^{3 *}$, Nivaldo B. Lima-Junior ${ }^{3}$, Francisco C.A. \\ Aguiar-Júnior ${ }^{4}$ e Katharine R.P. Santos ${ }^{4}$
}

\begin{abstract}
Silva M.C.R., Arandas M.J.G., Lima-Junior N.B., Aguiar-Júnior F.C.A. \& Santos K.R.P. 2016. [Histomorphometric analysis of cardiomyocytes and collagen deposition in the heart muscle of ovariectomized rats.] Análise histomorfométrica dos cardiomiócitos e deposição de colágeno no músculo cardíaco de ratas ooforectomizadas. Pesquisa Veterinária Brasileira 36(3):216-220. Área de Morfologia, Departamento de Morfologia e Fisiologia Animal, Universidade Federal Rural de Pernambuco, Rua Dom Manoel de Medeiros s/n, Dois Irmãos, Recife, PE 52171-900, Brazil. E-mail: julianaarandas@hotmail.com

This study aimed to evaluate the effect of oophorectomy on the morphometric variables of cardiomyocytes and quantification collagen in Wistar rats. Twenty rats were used and divided into two groups: GI - ovariectomized and GII - sham-ovariectomized. After recovery from anesthesia the animals were housed in separate cages and under the conditions of light / dark cycle $12 / 12 \mathrm{~h}$ for a period of six months. After the experimental period, the animals were anesthetized, the heart removed and immersed in $10 \%$ formaldehyde. The fragments of the ventricles were subjected to histological analysis and stained with hematoxylin and eosin. Histomorphometric analysis (cell area and nuclear area and volume) were performed by light microscopy and ImageJ software version 1.44. The data were submitted to ANOVA and when significant, complemented by Student's t test $(\mathrm{p}<0.001)$. There was a significant decrease in cellular and nuclear areas of the GI cardiomyocytes compared to GII and the area occupied by collagen was greater in cardiac muscle GI, when compared to GII. Therefore, it is concluded that the changes observed in the GI group cardiomyocytes, suggest a higher apoptotic activity in this group, due to decreased serum estrogen levels caused by ovariectomy and increased area occupied by collagen in oophorectomized group is associated the cardioprotective effect of estrogen.
\end{abstract}

INDEX TERMS: Histomorphometric analysis, heart muscle, rats, apoptosis, estrogen, early menopause, cardiac myocytes and collagen.

RESUMO.- Esse estudo objetivou avaliar o efeito da ooforectomia sob os parâmetros histomorfométricos dos cardiomiócitos e quantificação de colágeno em ratas Wistar. Vinte ratas foram utilizadas e separadas em dois grupos: GI - ooforectomizada e GII - sham-ooforectomizada. Após a recuperação anestésica os animais foram acomodados

\footnotetext{
${ }^{1}$ Recebido em 3 de julho de 2015.

Aceito para publicação em 14 de dezembro de 2015.

${ }^{2}$ Enfermeira, Centro Acadêmico de Vitória (CAV), Universidade Federal de Pernambuco (UFPE), Rua Alto do Reservatório s/n, Bela Vista, Vitória de Santo Antão, PE 55608-680, Brasil.

${ }^{3}$ Área de Morfologia, Departamento de Morfologia e Fisiologia Animal, UFRPE, Rua Dom Manoel de Medeiros, s/n, Dois Irmãos, Recife, PE 52171900, Brasil. *Autor para correspondência: julianaarandas@hotmail.com

${ }^{4}$ Núcleo de Biologia, CAV-UFPE, Rua Alto do Reservatório s/n, Bela Vista, Vitória de Santo Antão, PE 55608-680, Brasil.
}

em gaiolas e separados nas condições de ciclo claro/escuro $12 / 12 \mathrm{~h}$ por um período de 6 meses. Passado o período experimental, os animais foram anestesiados, o coração retirado e mergulhado em formaldeído a $10 \%$. Os fragmentos dos ventrículos foram submetidos ao processamento histológico e corados com hematoxilina e eosina. As análises histomorfométricas (área celular e área e volume nuclear) foram realizadas pelo microscópio de luz e software ImageJ versão 1.44. Os dados foram submetidos à análise ANOVA e quando significantes, complementados pelo teste $t$ de student $(\mathrm{p}<0,001)$. Observou-se uma diminuição significativa das áreas celular e nuclear dos cardiomiócitos do grupo GI quando comparado ao grupo GII, bem como a área ocupada, pelo colágeno, no músculo cardíaco foi maior em GI, quando comparado a GII. Diante disso, conclui-se que as alterações observadas nos cardiomiócitos do grupo GI, suge- 
rem uma maior atividade apoptótica nesse grupo, devido à diminuição dos níveis séricos do estrógeno provocado pela ooforectomia e o aumento da área ocupada pelo colágeno, no grupo ooforectomizado, está associada ao efeito cardioprotetor do estrógeno.

TERMOS DE INDEXAÇÃO: Histomorfométria, coração, ratas, apoptose, estrogênios, menopausa precoce, miócitos cardíacos, colágeno.

\section{INTRODUÇÃO}

A menopausa é definida como sendo a última menstruação, consequente à perda da função folicular ovariana ou à remoção cirúrgica dos ovários, provocando alterações hormonais no equilíbrio entre estrógeno e progesterona o que ocasiona inúmeras mudanças no organismo feminino. Este desequilíbrio pode acarretar danos fisiológicos, principalmente pela diminuição dos níveis séricos de estrógeno, provocando atrofia urogenital e declínio cognitivo, assim como, aumento do risco de doenças crônico-degenerativas, que podem manifestar-se a curto, a médio e em longo prazo. (Casanova \& Spritzer 2007, Ceravolo et al. 2007)

Atualmente, muitos estudos indicam os efeitos causados ao organismo, devido à ausência do estrógeno, porém poucos são os trabalhos voltados para as alterações histológicas no tecido cardíaco. Alguns defendem que o estrógeno tem a capacidade de agir diretamente nos miócitos cardíacos, por meio da ação modulatória negativa, na expressão gênica da proteína dos canais de cálcio $\mathrm{Ca}^{+}$tipo $\mathrm{L}$, controlando a excitabilidade e contratilidade da membrana dos cardiomiócitos, o que leva a uma redução no risco de arritmias, assim como de outras doenças cardiovasculares (Johnson et al. 1997). Podendo atenuar o desenvolvimento da hipertrofia cardíaca por meio da expressão aumentada do peptídeo natriurético atrial, mediante sobrecarga de pressão (Van Eickels et al. 2001).

As mulheres apresentam uma incidência aumentada de hipertrofia ventricular esquerda na pós-menopausa (Agabiti 2002). Além disso, outros efeitos do estrogênio são os envolvidos na síntese do colágeno e da elastina, através da modulação na expressão de seus genes e enzimas, reduzindo sua formação na matriz extra celular e aumentando a vascularização colateral, melhorando assim a perfusão tecidual, a longo prazo (Gerhard \& Ganz 1995, Sullivan 1996).

Tendo em vista esses aspectos e diante das informações constantes na literatura acerca dos efeitos do estrógeno sobre o tecido cardíaco, faz-se necessário um estudo mais aprofundado sobre as alterações histofisiológicas que a menopausa pode provocar ao coração. Nesse sentido, objetivou-se avaliar o efeito da ooforectomia sob os parâmetros histomorfométricos dos cardiomiócitos e quantificação de colágeno em ratas Wistar.

\section{MATERIAL E MÉTODOS}

Foram utilizadas 20 ratas de linhagem Wistar (Rattus norvegicus albinus), adultas jovens com 2 meses de idade, pesando entre $100 \mathrm{~g}$ e $200 \mathrm{~g}$, oriundas do Biotério do Departamento de Nutrição da Universidade Federal de Pernambuco (UFPE). Durante o experimento os animais foram mantidos em gaiolas plásticas com leito de maravalha no Biotério do Centro Acadêmico de Vitória CAV/
UFPE, com temperatura ambiente controlada a $22^{\circ} \mathrm{C}$ e iluminação artificial obtida com lâmpadas fluorescentes (modelo luz do dia de 40 Watts), com fotoperíodo claro e escuro, recebendo água filtrada e ração para ratos ad libitum.

Após dez dias, os animais com ciclos estrais regulares foram incluídos no estudo. Sendo distribuídos aleatoriamente entre dois grupos: GI (animais ooforectomizados) e GII (animais sham-ooforectomizados), e submetidos a ooforectomia ventral, segundo as normas de vivisecção de animais descritas pelo Colégio Brasileiro de Experimentação Animal (COBEA), sob a lei no 6638, de 8 de maio de 1979. O biológico foi aprovado pela Comissão de Ética e de Uso Animal (CEUA) do Centro de Ciências Biológicas (CCB) da UFPE (parecer no 23076.014664/2014-81).

Dez minutos antes do procedimento cirúrgico foi realizada a medicação antibiótica (Ampicilina sódica) $20 \mathrm{mg} / \mathrm{kg}$ por via intraperitoneal. A ooforectomia foi realizada com os animais pertencentes a GI após anestesia com $0,1 \mathrm{ml}$ de xilazina $(20 \mathrm{mg} / \mathrm{ml})$ e quetamina $(50 \mathrm{mg} / \mathrm{ml})$ por 100 gramas de peso, por via intraperitoneal. Os animais foram posicionados lateralmente para a localização dos ovários, o qual se distância $1 \mathrm{~cm}$ da coluna vertebral e $1 \mathrm{~cm}$ abaixo da última costela. Foi realizada tricotomia manual bilateralmente e antissepia com álcool $70 \%$ na região a ser incisada. Houve divulsão dos planos adiposo e muscular subjacente até a exposição do ovário. A ligadura foi realizada utilizando-se fio de nylon seguindo a remoção bilateral dos ovários. Em seguida, os órgãos removidos foram fixados em formol tamponado a 10\% e processados seguindo a técnica histológica de rotina. Os animais do grupo GII passaram pelos mesmos procedimentos cirúrgicos descritos acima, porém não sofreram remoção bilateral dos ovários.

No período pós-operatório os animais de ambos os grupos foram colocados em gaiolas isoladas para a recuperação anestésica, com administração de dipirona $200 \mathrm{mg} / \mathrm{kg}$ por via oral em dose única. Após a fase pós-operatória, os animais foram acomodados em gaiolas e separados nas condições de ciclo claro/escuro $12 / 12 \mathrm{~h}$ por um período de 6 meses.

Ao término do período experimental, os animais foram novamente anestesiados e após toracotomia, o coração foi removido, ainda com batimentos, sendo imediatamente seccionado perpendicularmente ao seu maior eixo separando a base do ápice, ao nível dos ventrículos e mergulhado em formaldeído a $10 \%$ neutro tamponado (NBF) por um período de $24 \mathrm{~h}$, para posterior processamento histológico. Os corações foram incluídos em blocos de parafina de tal maneira que fosse possível realizar cortes transversais nos ventrículos. Desses blocos, foram obtidos cortes de $4 \mu \mathrm{m}$, os quais foram corados por hematoxilina e eosina (HE), e picrossirius para marcação do colágeno. Ao final da retirada dos corações, os animais foram descartados segundo as normas vigentes na UFPE/CAV.

As análises quantitativas e morfológicas foram realizadas no Laboratório de Biotecnologia e Fármacos do Centro Acadêmico de Vitória/UFPE. Para avaliação morfológica utilizamos microscópio de luz (Nikon Eclipse E200). Para a obtenção dos dados morfométricos, imagens de cortes transversais e longitudinais de feixes musculares do ventrículo esquerdo foram capturadas através de uma câmera de alta resolução (Moticam 2300) adaptada a microscópio de luz (Nikon Eclipse E200) que foram transmitidas ao computador.

Para a avaliação das áreas celulares e nucleares dos cardiomiócitos foram obtidas 20 fotomicrografias de cada coração, perfazendo um total de 200 imagens por grupo. Posteriormente, as fotomicrografias foram analisadas através do software ImageJ versão 1.44 (Research Services Branch, U.S. National Institutes of Health, Bethesda, MD, USA.), sendo medidas as áreas de 50 células e seus respectivos núcleos por imagem no corte transversal, já no 
corte longitudinal foi medido o volume nuclear através do maior e do menor diâmetro de 50 núcleos por imagem, sendo os valores aplicados na seguinte fórmula: $v=a 2 . b / 1,91$ onde $a=$ menor diâmetro, b=maior diâmetro e 1,91 é uma constante. Em seguida foi realizada a quantificação de colágeno, através do percentual da área composta por colágeno. A análise estatística foi realizada com o auxílio do programa SPSS 15.0 (Statistical Package for Social. Sciences).

Os dados obtidos foram submetidos à análise de variância (ANOVA) e quando significantes, complementados pelo teste t de student, para observar as possíveis diferenças entre os grupos. 0 nível de significância foi fixado em 0,05 ou $5 \%(\mathrm{p} \leq 0,05)$.

\section{RESULTADOS}

A análise histológica revelou que os cardiomiócitos de ambos os grupos apresentaram organização estrutural típica, com células ramificadas contendo um a dois núcleos centralizados. Em cortes transversais foi possível verificar delicadas bainhas de tecido conjuntivo envolvendo as fibras musculares, que veiculam vasos sanguíneos e nervos (Fig.1).

A análise morfométrica mostrou que tanto a área celular quanto a área nuclear dos cardiomiócitos do GI (animais ooforectomizados) foram menores quando comparado ao
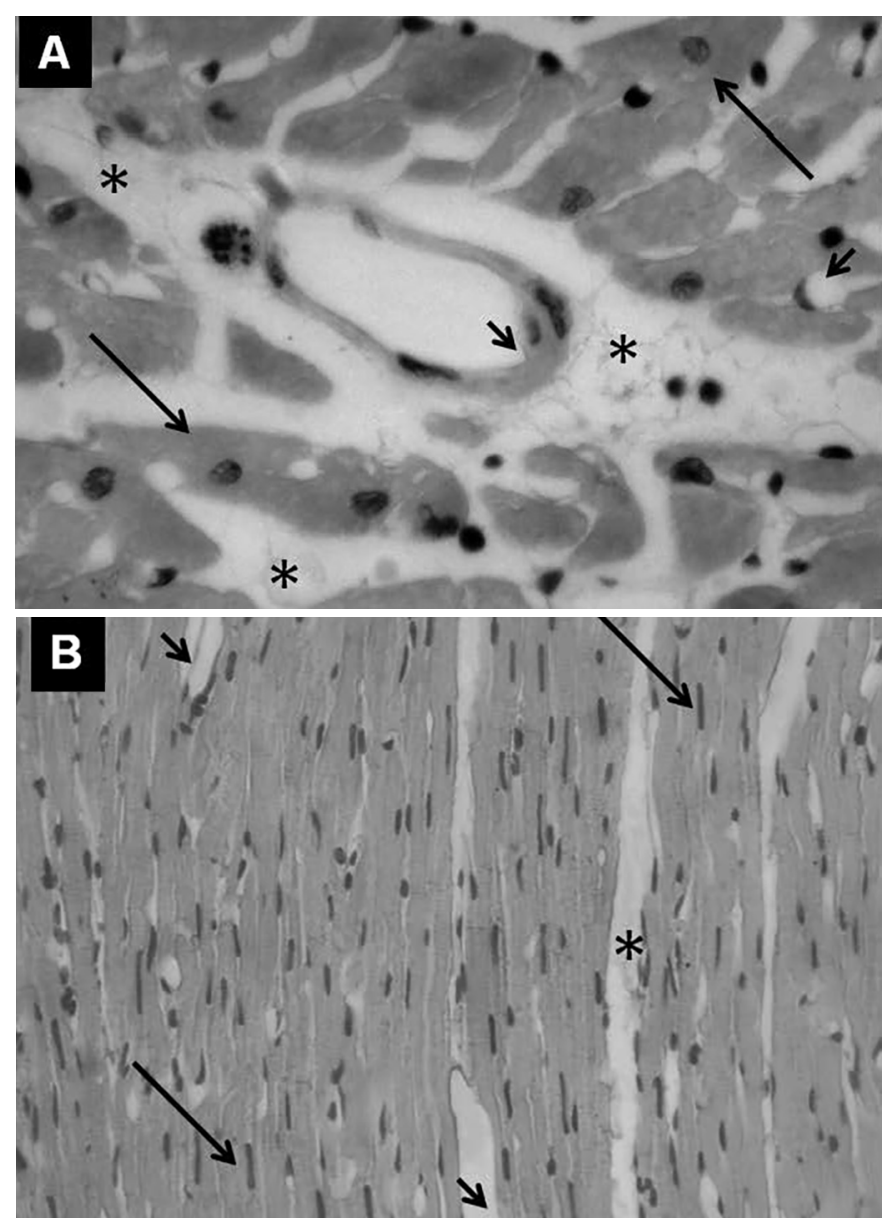

Fig.1. (A) Ventrículo esquerdo em corte transversal de GI (ooforectomizada). (B) Ventrículo esquerdo em corte longitudinal de GII (sham-ooforectomizada). Setas longas: cardiomiócitos com núcleo centralizado. Seta curta: vaso sanguíneo. $\left({ }^{*}\right)$ : Tecido conjuntivo. HE, obj.40x.

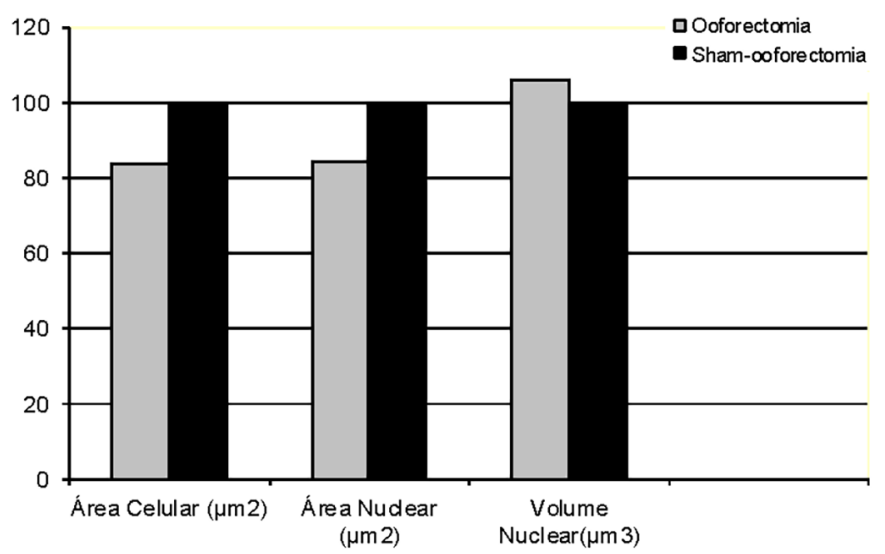

Fig.2. Valor médio percentual das áreas celular (82,82\%) e nuclear $(84,26 \%)$ do grupo ooforectomizado em comparação com o grupo sham-ooforectomizado (100\%). O volume nuclear do grupo sham-ooforectomizado foi menor que o grupo ooforectomizado em $5,89 \%$.

GII (animais sham-ooforectomizados), sendo estatisticamente significativas (Fig.2). Enquanto que o volume nuclear foi maior para os cardiomiócitos do GI, entretanto não foi significativo.

Em relação à área ocupada pelo colágeno foi verificado que o GI apresentou maior área, quando comparado ao GII, sendo essa medida estatisticamente significativa (valor de $p=0,001)$. Esses resultados encontram-se expressos no Quadro 1.

Quadro 1. Média e desvio padrão, da área ocupada pelo colágeno, no coração de ratas dos grupos Ooforectomizado (GI) e Sham-ooforectomizado (GII)

\begin{tabular}{lcc}
\hline \multicolumn{1}{c}{ Grupo } & Média & Desvio padrão \\
\hline Ooforectomizado (GI) & 1,3803 & 1,28656 \\
Sham-ooforectomizado (GII) & 0,7956 & 0,69785
\end{tabular}

Valor de $\mathrm{p}=0,001$.

\section{DISCUSSÃO}

Os resultados obtidos com o presente estudo evidenciam uma diminuição das áreas celular e nuclear dos cardiomiócitos do GI, sugerindo uma maior atividade apoptótica neste grupo, uma vez que a célula em apoptose modifica suas características morfológicas, o que acontece de modo bastante rápido. E essas alterações são evidenciadas tanto a nível celular quanto nuclear como: a retração de forma que causa a perda da aderência com a matriz extracelular e com as células vizinhas, a condensação da cromatina concentrada próximo a membrana nuclear, que por vez se mantém intacta. A membrana celular forma prolongamentos, o núcleo decompõe-se e estes prolongamentos se rompem formando sacos com conteúdo celular, denominados de corpos apoptóticos. Estes corpos são eficazmente fagocitados por macrófagos e retirados sem que ocorra reação inflamatória no local (Ziegler \& Groscurth 2004).

Em ratos, estrogênios atenuam a hipertrofia do miocárdio, o que resulta na diminuição dos índices de apoptose e necrose dos cardiomiócitos, o que relaciona aos resultados desse estudo, visto que a ooforectomia e, por conseqüência, 
o declínio dos estrógenos, induziu a diminuição das áreas celular e nuclear dessas células, indicando uma maior atividade apoptótica (Liou et al. 2010).

É conhecido na literatura atual que o estrógeno é capaz de regular a expressão gênica através da ligação e interação a receptores de estrogênio (ER) clássicos, como o ER $\alpha$ (estrogen receptor alpha) e ER $\beta$ (estrogen receptor beta) presentes no núcleo dos cardiomiócitos, que atuam como fatores transcricionais operados por ligantes que se unem ao DNA com auxilio de co-ativadores e co-repressores, alterando assim, a transcrição da fita dupla (Murphy 2011). 0 efeito do estrógeno pela regulação dos genes em ER $\alpha$ e ER $\beta$ pode variar de acordo com a diferença da ligação de co-ativadores e co-repressores (Kararigas et al. 2012).

Outros estudos evidenciam que o estrogênio também pode se ligar a seus receptores presentes na membrana plasmática e ativar vias de sinalização. 0 ER $\alpha$ tem demonstrado estar também associado com a membrana plasmática, e a ligação do estrógeno é importante para ativar a via de sinalização do fosfoinositol-3-quinase (PI3K) (Kim et al. 2011). A via PI3K leva a ativação de AKt (proteína quinase B ou PBK) é fundamental para sinalização de sobrevivência celular, pois quando ativado fosforila e inibe os pró apoptóticos B-cell lymphoma 2 (Bcl-2) e a proteína X (Bax) (Liou et al. 2010). A Bcl-2 faz parte de uma família de proteínas que participa ativamente da regulação da apoptose, podendo reprimi-la por impedir a liberação do citocromo $c$, ou induzi-la. Já a Bax, é uma proteína pró apoptótica (Hengartner, 2000). Estudos demonstraram que Bcl-2 e Bax possuem a capacidade de se unir formando homodímeros (Bcl-2-Bcl2 e Bax-Bax) e heterodímeros (Bcl-2-Bax), sendo que o equilíbrio entre estes podem definir o balanço pró apoptótico ou anti-apoptótico na célula (Petros et al. 2004).

Por outro lado, esse estudo também elucidou uma maior quantidade de deposição do colágeno no músculo cardíaco do grupo ooforectomizado (GI) e uma menor quantidade no grupo controle sham-ooforectomizado (GII). Esses resultados são semelhantes ao encontrado no estudo de Cabanelas et al. (2012).

Esse aumento da deposição de colágeno em GI pode ser compreendido e relacionado à associação existente entre o estrógeno e a musculatura cardíaca, já que esse hormônio apresenta efeitos cardioprotetores sincronizado diretamente com o desempenho desse músculo. (Pines et al. 1993, Schierbeck et al. 2012). Com relação a esses efeitos cardioprotetores do estrógeno, destaca-se a sua atuação no metabolismo das lipoproteínas. Assim contribui no aumento da produção de HDL (lipoproteína de alta densidade) e, em contrapartida, atua na degradação de LDL (lipoproteína de baixa densidade) (Mendelsohn \& Karas 1999a).

Diante disso, essas modificações podem ser ocasionadas devido ao remodelamento do músculo cardíaco que, caso não seja adaptado, pode gerar um risco no desenvolvimento de doenças cardiovasculares. Sendo assim, os estrogênios são agentes protetores contra a fibrose cardíaca. Além disso, outros trabalhos também dão suporte a esse mesmo raciocínio, visto que a reposição $17 \beta$-estradiol diminui a deposição de colágeno tipo I no miocárdio de ra- tas, impedindo a fibrose perivascular (Xu et al. 2003, Voloshenyuk et al. 2010, Cabanelas et al. 2012).

Dessa forma, como o estrógeno apresenta esse efeito cardioprotetor, a hipertrofia do músculo cardíaco pode ocasionar a fibrose, devido à insuficiência desse hormônio, cuja distribuição no organismo foi interrompida pela ooforectomia. Sendo que a fibrose interfere diretamente na excitação-contração das células encontradas no músculo cardíaco (cardiomiócitos) durante os processos de sístole e diástole, causando rigidez e dificultado, em consequência, o débito cardíaco. Portanto, a elevação na expressão dos genes de colágeno é característica de ser encontrada no miocárdio que apresenta hipertrofia e, consequentemente, a fibrose cardíaca (Manabe et al. 2002, Berk et al. 2007, Pedram et al. 2008).

\section{CONCLUSÃO}

Concluímos que as alterações ocorridas nos cardiomiócitos do grupo ooforectomizado, indicam uma maior atividade apoptótica neste grupo. Isso pode estar relacionado à diminuição dos níveis séricos do estrógeno, por conta da ooforectomia. Por outro lado, o aumento da área ocupada pelo colágeno, nesse mesmo grupo, está associado ao efeito cardioprotetor que o estrógeno exerce sobre o músculo cardíaco.

\section{REFERÊNCIAS}

Agabiti R.E. 2002. Left ventricular hypertrophy and heart failure in women. J. Hypertens. 20(Suppl.2):34-38.

Berk B.C., Fujiwara K. \& Lehoux S. 2007 ECM remodeling in hypertensive heart disease. J. Clin. Invest. 117:568-575.

Cabanelas L.A., Carbonel A.A.F., Santos M.A., Simões R.S., Liberatori-Filho A.W., Baracat E.C. \& Soares Júnior J.M. 2012. Morfologia dos cardiomiócitos e quantificação do colágeno no miocárdio de ratas tratadas com isoflavonas ou estrogênios. Revta Bras. Ginecol. Obstet. 34(10):447-452.

Casanova G. \& Spritzer P.M. 2007. Aspectos fisiopatológicos: estrogênios, menopausa e terapia hormonal. Hipertensão 10(4):131-134.

Ceravolo G.S., Tostes R.C., Fortes Z.B. \& Carvalho M.H.C. 2007. Efeitos do estrógeno no sistema cardiovascular. Hipertensão 10(4):124-130.

Gerhard M. \& Ganz P. 1995. How do we explain the clinical benefits of estrogen? From bedside to bench. Circulation 92(1):5-8.

Hengartner M.0. 2000. The biochemistry of apoptosis. Nature 407(6805): 770-776.

Johnson B.D., Zheng W., Korach K.S., Scheuer T., Catterall W.A. \& Rubanyi G.M. 1997. Increased expression of the cardiac L-type calcium channel in estrogen receptor deficient mice. J. Gen. Physiol. 110(2):135-140.

Kararigas G., Bito V., Tinel H., Becher E., Baczko I., Knosalla C, Albrecht-Küpper B., Sipido K.R. \& Regitz-Zagrosek V. 2012. Transcriptome characterization of estrogentreated human myocardium identifies myosin regulatory light chain interacting protein as a sex-specific element influencing contractile function. J. Am. Coll. Cardiol. 59(4):410-17.

Kim K.H., Toomre D. \& Bender J.R. 2011. Splice isoform estrogen receptors as integral transmembrane proteins. Mol. Biol. Cell 22(22):4415-423.

Liou C.M., Yang A.L., Kuo C.H., Tin H., Huang C.Y. \& Lee S.D. 2010. Effects of $17 \beta$ estradiol on cardiac apoptosis in ovariectomized rats. Cell Biochem. Funct. 28(6):521-528.

Manabe I., Shindo T. \& Nagai R. 2002. Gene expression in fibroblasts and fibrosis: involvement in cardiac hypertrophy. Circ. Res. 91:1103-1113.

Mendelsohn M.E. \& Karas R.H. 1999. The protective effects of estrogen on the cardiovascular system. N. Engl. J. Med. 340(23):1801-1811.

Murphy E. 2011. Estrogen signaling and cardiovascular disease. Circ. Res. 109(6):687-696. 
Pedram A., Razandi M., Lubahn D., Liu J., Vannan M. \& Levin E.R. 2008. Estrogen inhibits cardiac hypertrophy: role of estrogen receptor- $\beta$ to inhibit calcineurin. Endocrinology 149(7):3361-3369.

Petros A.M., Olejniczak E.T. \& Fesik S.W. 2004. Structural biology of the Bcl2 family of proteins. Biochim. Biophys. Acta. 1644(2/3):83-94.

Pines A., Fisman E.Z., Levo Y., Drory Y., Ben-Ari E., Motro M. \& Ayalon D. 1993. Menopause-induced changes in left ventricular wall thickness. Am. J. Cardiol. 72(2):240-241.

Schierbeck L.L., Rejnmark L., Tofteng C.L., Stilgren L., Eiken P., Mosekilde L., Kober L. \& Jensen J.E. 2012. Effect of hormone replacement therapy on cardiovascular events in recently postmenopausal women: randomized trial. BMJ 345:e6409.

Sullivan J.M. 1996. Practical aspects of preventing and managing athe- rosclerotic disease in post-menopausal women. Eur. Heart J. 17(Suppl D):32-37.

Van Eickels M., Groiif C., Cleutjens J.P., Jassen B.J., Wellens H.J. \& Doevendans P.A. 2001. 17(beta)estradiol attenuates the development of pressure overload hypertrophy. Circulation 104(12):1419-423.

Voloshenyuk T.G. \& Gardner J.D. 2010. Estrogen improves TIMP-MMP balance and collagen distribution in volume-overloaded hearts ofovariectomized females. Am. J. Physiol. Regul. Integr. Comp. Physiol. 299(2):R683-693.

Xu Y., Arenas I.A., Armstrong S.J. \& Davidge S.T. 2003. Estrogen modulation of left ventricular remodeling in the aged heart. Cardiovasc. Res. 57(2):388-394.

Ziegler U. \& Groscurth P. 2004. Morphological features of cell death. News Physiol. Sci. 19:124-128. 